\title{
Forward to the Special Issue on Hox/Tale Transcription Factors in Development and Disease
}

\author{
Deneen M. Wellik, ${ }^{1 *}$ Miguel Torres, ${ }^{2}$ and Marian A. Ros $^{3}$
}

Submitted 4 October 2013; Accepted 11 October 2013

\section{INTRODUCTION}

This special issue of Developmental Dynamics was inspired by the $H O X$ and TALE Transcription Factors in Development and Disease COST Action that has been meeting annually since 2009. This action has gathered researchers from Spain, France, Switzerland, Portugal, Italy, Germany, Netherlands, Hungary, Sweden, Denmark, Norway, Australia, United Kingdom, Canada, Russia, Israel, Belgium, Poland, Turkey, and the United States and focuses on the highly conserved HOX and TALE transcription factors. HOX and TALE factors occupy a central position in several areas of biomedical research. They are important in the developmental patterning of numerous organ systems and serve as key models for the study of numerous developmental processes, transcriptional mechanisms, epigenetic control of gene expression, and the balance between proliferation and differentiation.

The most established roles for Hox genes across multiple animal species are in specifying regional identity along the anteroposterior axis of the body and along the proximodistal axis of the limb. The way anatomy correlates with the genomic "landscape" of the Hox clusters and how this is translated in the control of morphogenesis has fascinated scientists for decades. These aspects of Hox patterning and the mechanisms by which these patterning processes occur are discussed in several of the reviews in this issue. The Pbx, Prep, and Meis group of transcription factors, which belongs to the TALE family of homeodomain proteins, are the classic cofactors of Hox proteins. However, this gene family also appears to have broader Hox-independent roles and both aspects of their function are reviewed in this issue. Together, these transcription factors comprise one of the most evolutionarily conserved and broadly used sets of gene regulation modules in the animal kingdom. Importantly, growing evidence shows that this regulatory network is essential to maintain tissue homeostasis during adult life in various organs and tissues, and their improper function leads to many disease processes in addition to their well-established involvement in leukemias. This collection of reviews highlights several important areas of current focus in our continuing quest to understand the mechanisms of their regulation and function.

Along with the more longstanding view of the critical role for Hox transcription factors in axial patterning, recent work in vertebrates has shown that they also cooperate with other transcription factors and signaling pathways in axial extension. Caudal homeodomain proteins, the Cdx transcription factors, are important regulators of the maintenance of Wnt expression, and loss of these genes leads to dramatic disruptions in axial extension. As expected, these defects can be rescued by forced expression of Wnt. However, they also can be rescued by forced expression of at least some "trunk" Hox genes, demonstrating that Hox genes can function in this pathway. Remarkably, the final group of Hox genes, the Hox13 paralogs, is antagonistic to this activity so that their precocious activation in the posterior growth zone leads to early truncation of the axis. Evidence suggests the truncating activity reflects an endogenous role in terminating axial extension in vertebrates. Neijts et al., review how Hox gene function can be currently synthesized with

\footnotetext{
${ }^{1}$ Department of Internal Medicine, Division of Molecular Medicine and Genetics, University of Michigan, Ann Arbor, Michigan

${ }^{2}$ Departamento de Desarrollo y Reparación Cardiovascular, Centro Nacional de Investigaciones Cardiovasculares, Madrid, Spain

${ }^{3}$ Instituto de Biomedicina y Biotecnología de Cantabria (CSIC-UC-SODERCAN) and University of Cantabria, Santander, Spain

*Correspondence to: Deneen M. Wellik, Department of Internal Medicine, Division of Molecular Medicine and Genetics,

University of Michigan, Ann Arbor, MI 48109-2200. E-mail: dwellik@umich.edu
}

DOI: $10.1002 /$ dvdy.24098

Published online 21 November 2013 in Wiley Online Library (wileyonlinelibrary.com). 
existing genetic and embryologic data on axial extension in vertebrates (Neijts et al., this issue).

With regard to understanding how Hox proteins function, strides continue to be made in many areas of organogenesis in the developing embryo and in their relevance to disease. During the past two decades, many Hox genes along the cluster have been shown to play important roles in the early patterning of both the hindbrain and the spinal cord (reviewed previously in Narita and Rijli, 2009; Tumpel, et al., 2009). In this series, Di Bonito et al. highlight progress in the field related to the formation of region-specific sensorimotor circuit formation. This patterning activity occurs later in development than the previously appreciated role in hindbrain patterning, but is clearly important for achieving the high complexity of integration observed in neural circuitry. The authors share their views on how early neural tube segmentation and later circuitry specifications may be related.

Moving to the other extremity of the developing animal, Brison et al. (this issue) review our current understanding of the role of Hoxd13 in digit polysyndactyly phenotypes in humans. A relatively broad range of autopod phenotypes is reported in human syndromes associated with mutations in Hoxd13 in humans. This review focuses on what we have learned and continue to learn from using both mouse and chicken as model organisms to understand the effects of various Hoxd13 mutations, and how these studies have informed human pathologies.

It is impossible to discuss the role of Hox genes in patterning processes without considering the mechanism by which they are coordinately expressed. Many reports illustrate how timing affects the anterior limit of expression of the Hox transcription factors. In Casaca et al. (this issue), the importance of timing of expression on Hox boundary formation is reviewed. Changing the timing of expression onset by manipulating regulatory regions in the locus, either transgenically or by changing the epigenetic control of the cluster, results in phenotypic changes along the axial skeleton. miRNAs produced at the
Hox genomic loci may also provide additional levels of control, and recent evidence also suggests that ribosomal proteins may contribute to Hox gene expression. This idea is explored by Soshnikova (this issue) in a review of the mechanisms by which dynamic histone marks along the clusters and higher-order chromatin structure determine Hox gene regulation. The involvement of noncoding RNAs in regulating the clusters in vitro and in vivo are also discussed, and these studies are put into the context of more general transcriptional regulatory mechanisms.

To date, a myriad of studies have highlighted various domains and motifs within the Hox proteins that control their functional properties. One important limit of our understanding of Hox function in development and disease is the lack of information regarding novel transcriptional partners in addition to the TALE family of factors that drive downstream target gene expression. Casaca et al. (this issue) reviews several examples of non-Hox-TALE enhancers that are used by multiple Hox proteins to drive expression in opposing directions. There are a small but growing number of examples of these bona fide Hox-regulated enhancers that do not cooperate with TALE proteins to affect gene regulation. In at least two important examples published by three laboratories, Pax proteins appear to act as co-factors with Hox factors in differential gene regulation (Gong et al., 2007; Plaza et al., 2008; Yallowitz et al., 2009; Guerreiro et al., 2013). Current findings are reviewed, although continuing work in the field will determine whether this represents another general mechanism.

Although there are many features of Hox proteins that make them particularly difficult to study, many in the field would agree that there are two main impediments toward making progress along these fronts. First, all Hox homeodomains (DNA binding motifs) recognize the highly similar or identical recognition sequence TAAT. The exceedingly low amount of information in this consensus sequence (occurring at random theoretically every 128 base pairs in any genome) precludes the use of bioinformatic approaches to identify putative enhancer sites. Some Hox-
TALE joint enhancer motifs have been identified, but Hox-regulated sites that do not rely on cooperative binding with TALE proteins cannot be identified bioinformatically. This handicap would be at least somewhat alleviated by the ability to perform high-quality molecular biochemistry using antibodies in vivo and in cell culture; however, these reagents have been exceptionally difficult to generate, likely due to the very high degree of identity among orthologous Hox proteins from even divergent mammals. Two reviews in this issue address this problem. Ladam et al. (this issue) present a broad and comprehensive review of the existing literature, including clearly documented interactions with the TALE cofactors as well as newer evidence that many additional components are involved in both repression and activation from Hoxregulated enhancer regions. The most recent views on how these enhancer sites switch from activation to repression is discussed. The focused review presented by Merabet and Dard (this issue) surveys the very latest methodologies that may allow for global explorations of binding partners. Using a combination of high-throughput mass spectrometry and single-, double-, and tripletagging proteins, the authors are optimistic that deciphering Hox interaction networks on a more comprehensive scale may soon be possible. Through an in-depth review of the literature and databases, Rezsohazy (this issue) also surveys available information on numerous additional Hox cofactors, focusing on the possible involvement of Hox proteins in nontranscriptional processes through the interaction with these new identified cofactors.

This issue is complimented by reviews on the TALE factors themselves. Longobardi et al. (this issue) offer a comprehensive review of $\mathrm{Pbx}$, Meis, and Prep subfamilies, including their structure, interactions, and binding preferences in vitro and in vivo. Members from these three sets of proteins form dimers with one another, in some instances in collaboration with Hox proteins. Additionally, genomewide DNA-binding explorations demonstrate sets of binding sites in the genome that both require and are 
independent of Hox. Taken together, some general rules of transcriptional regulation have been generated and are reviewed in this contribution.

Finally, the review by Schulte and Dale (this issue) highlights the many roles for TALE factors in determining cell fate specification and differentiation in the developing nervous system of vertebrates and invertebrates, the factors upstream and downstream of these TALE factors, and the overlap (and lack thereof) with Hox in these systems. Authors present the current knowledge about how TALE proteins function to modulate neuronal cell fate and discuss further challenges in this field.

As this field continues to move forward, the combination of more sophisticated tools for genome- and systems-wide analyses, along with more specific genetic and molecular explorations with respect to initial body patterning, organogenesis, and disease will hopefully allow significant new progress in determining the downstream mechanisms of Hox and TALE function. An important task ahead is to accurately determine the extent and nature of Hox and TALE cooperative and independent functions. With new studies continuing to highlight roles for both of these sets of genes in adult homeostasis, repair, and disease states, including cancers, it is clear that we are closer to the beginning than to the end of these stories.

\section{REFERENCES}

Gong KQ, Yallowitz AR, Sun H, Dressler GR, Wellik DM. 2007. A Hox-Eya-Pax complex regulates early kidney developmental gene expression. Mol Cell Biol 27:761-768.
Guerreiro I, Nunes A, Woltering JM, Casaca A, Nóvoa A, Vinagre T, Hunter ME, Duboule D, Mallo M. 2013. Role of a polymorphism in a Hox/Pax-responsive enhancer in the evolution of the vertebrate spine. Proc Natl Acad Sci U S A 110:10682-10686.

Narita Y, Rijli FM. 2009. Hox genes in neural patterning and circuit formation in the mouse hindbrain. Curr Top Dev Biol 88:139-167.

Plaza S, Prince F, Adachi Y, Punzo C, Cribbs DL, Gehring WJ. 2008. Crossregulatory protein-protein interactions between Hox and Pax transcription factors. Proc Natl Acad Sci U S A 105: 13439-13444.

Tumpel S, Wiedemann LM, Krumlauf R. 2009. Hox genes and segmentation of the vertebrate hindbrain. Curr Top Dev Biol 88:103-137.

Yallowitz AR, Gong KQ, Swinehart IT, Nelson LT, Wellik DM. 2009. Nonhomeodomain regions of Hox proteins mediate activation versus repression of Six2 via a single enhancer site in vivo. Dev Biol 335:156-165. 